\title{
Long-Term Prognostic Predictors of Esophageal Squamous Cell Carcinoma Potentially Indicated for Endoscopic Submucosal Dissection
}

\author{
Tomohiko Suzuki $^{a}$ Kazuhiro Furukawa ${ }^{a}$ Kohei Funasaka ${ }^{a}$ b Eri Ishikawa ${ }^{a}$ \\ Tsunaki Sawadac Keiko Maedac Takeshi Yamamurac Takuya Ishikawa ${ }^{c}$ \\ Eizaburo Ohno $^{a}$ Masanao Nakamura $^{\mathrm{a}}$ Hiroki Kawashimac ${ }^{\mathrm{R}}$ Roji Miyahara ${ }^{\mathrm{a}}$ \\ Mitsuhiro Fujishiro ${ }^{a}$ \\ aDepartment of Gastroenterology and Hepatology, Nagoya University Graduate School of Medicine, Nagoya, Japan; \\ ${ }^{b}$ Department of Gastroenterology, Fujita Health University School of Medicine, Toyoake, Japan; 'Department of \\ Endoscopy, Nagoya University Hospital, Nagoya, Japan
}

\section{Keywords}

Esophageal squamous cell carcinoma - Endoscopic

submucosal dissection · Comorbidity

\begin{abstract}
Introduction: Patients with esophageal squamous cell carcinoma (ESCC) have various comorbidities. Thus, it is necessary to determine the appropriateness of performing treatment based on the patient's general condition. Aim: This study aimed to clarify the prognostic predictors of ESCC indicated for endoscopic submucosal dissection (ESD). Methods: This retrospective study enrolled 241 patients with superficial ESCC endoscopically diagnosed as ESD-indicated lesions at the Nagoya University Hospital between January 2007 and December 2017. We evaluated the 3- and 5-year overall survival (OS) rates and prognostic predictors, such as the Prognostic Nutritional Index (PNI), Charlson Comorbidity Index (CCl), Psoas Muscle Index, and Controlling Nutritional Status score. Furthermore, we created a score-based classification using the prognostic predictors identified by multivariate analysis, and the 3- and 5-year OS rates were compared among the calculated scores. Results: In the multivariate analysis, $\mathrm{PNI}<45$ (hazard ratio [HR]:2.39; 95\% confidence
\end{abstract}

interval [Cl]: $1.28-4.46 ; p=0.006)$ and $\mathrm{CCl} \geq 3$ (HR: 4.42; 95\% Cl: $2.40-8.12 ; p<0.001)$ were significantly associated with the OS. Based on the HR, 0 and 1 were assigned to PNI and 0 , 2 , and 4 were assigned to $\mathrm{CCl}$, and the score classification of $0-5$ points was created. The 3 - and 5 -year OS rates in patients with a score 3 were significantly higher than in those with scores 4 and 5 . As a result of scoring, the prognosis was stratified; the 3- and 5-year OS rates in patients with scores 4 and 5 , that is, $\mathrm{CCl} \geq 6$, were clearly low, at approximately $10 \%$. Conclusions: $\mathrm{CCl}$ and $\mathrm{PNI}$ can be prognostic predictors of patients with superficial ESCC indicated for ESD. Observation without ESD might be an acceptable strategy among patients with $\mathrm{CCl} \geq 6$.

\footnotetext{
(c) 2020 The Author(s) Published by S. Karger AG, Basel
}

\section{Introduction}

In recent years, studies have attempted to predict prognosis based on the nutritional status and comorbidities of patients with gastrointestinal cancers. As typical prognostic predictors, Prognostic Nutritional Index (PNI), Controlling Nutritional Status (CONUT) score, Psoas Muscle Index (PMI), and Charlson Comorbidity

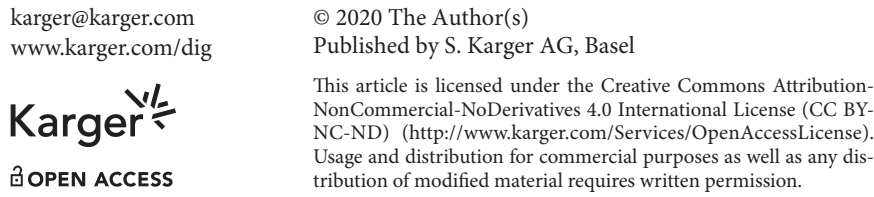

Kazuhiro Furukawa

Department of Gastroenterology and Hepatology Nagoya University Graduate School of Medicine 65 Tsurumai-cho, Showa-ku, Nagoya 466-8550 (Japan) kazufuru@med.nagoya-u.ac.jp 
Index (CCI) have been reported. The PNI is the nutritional index originally proposed to evaluate the surgical risks of patients with gastrointestinal cancer [1]. Recently, the PNI has been identified as prognostic predictors in patients with gastric cancer, pancreatic cancer, and hepatocellular carcinoma [2-4]. The CONUT score, which was calculated by scoring serum albumin level, lymphocyte count, and total cholesterol level, has also been reported to be associated with prognosis in gastrointestinal cancers [5-7]. PMI, which is calculated by iliopsoas muscle area at the third lumbar vertebrae level and body height, is an index used originally for a sarcopenia criterion in cirrhosis patients [8]. Recent studies revealed that PMI was associated with the long-term results of patients with CKD and pancreatic cancer $[9,10]$. CCI, which stratifies the risk of death from comorbidities, has been commonly used to evaluate the clinical outcomes, including prognosis and complications [11-13]. CCI is calculated as the total scores assigned to several comorbidities (e.g., congestive heart failure, myocardial infarction, cerebrovascular disease, diabetes mellitus, CKD, liver disease, solid tumor, leukemia, and AIDS) [14].

Endoscopic submucosal dissection (ESD) for esophageal squamous cell carcinoma (ESCC) can be performed safely with recent technological advances [15]. Short-term results were reported as $5.0-5.2 \%$ for perforation, $0-2.1 \%$ for bleeding, and $7.2-11.6 \%$ for stenosis $[16,17]$. As a long-term prognosis, the 5 -year overall survival (OS) rate after ESD has been reported to be $>85 \%$ [18-20]. However, it is necessary to determine the appropriateness of performing treatment based on the general condition, comorbidity, and nutritional condition, because it is reported that patients with ESCC are likely to have simultaneous and metachronous cancers in other organs as well as the esophagus [21-23]. Although there have been reports on the prognostic predictors of patients with advanced esophageal cancer and additional resection after esophageal ESD, there are no reports on the prognostic predictors of patients with ESCC potentially indicated for ESD $[24,25]$. To fill this research gap, this study aimed to clarify the prognostic predictors of ESCC indicated for ESD.

\section{Materials and Methods}

\section{Patients and Study Design}

In this single-center retrospective study, a total of 243 patients with superficial ESCC diagnosed as invasion depth of mucosa or shallow submucosa $(<200 \mu \mathrm{m})$ endoscopically and no metastasis by CT scan, for which ESD was potentially indicated, at the Nagoya University Hospital between January 2007 and December 2017 were enrolled [26, 27]. In this study, follow-up for 1 year or longer was defined as long-term follow-up. Two patients whose follow-up periods were within 1 year because of dropout were excluded from this study. In detail, 226 were ESD cases, 10 were observation cases, and 5 were chemoradiotherapy (CRT) cases. Depth of invasion was determined by white light endoscopy and magnifying endoscopy with narrow-band imaging or blue light imaging according to Inoue classification (before 2011) or Japanese Esophageal Society Classification (after 2012) [28, 29]. In patients who were treated multiple times at the Nagoya University Hospital, we chose the first lesion considered to be an index lesion. In our hospital, patients with all-round lesions were indicated for surgery during the study period; thus, they were excluded from the study population. Metastasis was determined using chest and abdominal CT in principle.

Age, sex, preference history (drinking, smoking), pathological results, treatment option, general condition (Eastern Cooperative Oncology Group performance status [ECOG PS], BMI), nutritional status (PNI, CONUT score, and PMI), and comorbidity (CCI) were divided into 2 or more groups, and their association with prognosis was examined. BMI was calculated as weight $(\mathrm{kg})$ divided by height (m) squared. The PNI was calculated as $10 \times$ serum albumin level (g/ $\mathrm{dL})+0.005 \times$ lymphocyte count $\left(/ \mathrm{mm}^{3}\right)$. ECOG PS was classified as PS $0-1$ and PS $\geq 2$. PNI was classified into 2 groups, namely, PNI $<45$ and $\mathrm{PNI} \geq 45$. The CONUT score was classified into 2 groups: normal CONUT $0-1$ and malnutrition CONUT $\geq 2$. Preference history, BMI, PMI, and CCI were classified into 2 groups using receiver operating characteristic (ROC) curve analysis. Furthermore, score classification was based on items extracted as prognostic predictors, which were identified by multivariate analysis, and the 3- and 5-year OS rates were compared among calculated scores.

This study complied with the Declaration of Helsinki and was approved by the Nagoya University Research Ethics Committee. The choice of treatment option was determined with sufficient informed consent.

\section{Data Collection}

Survival information of the enrolled patients was principally obtained from their medical records. In patients who had not been consulted since 2019, their survival or death was confirmed by sending a letter to their referral or home. The follow-up period was defined as the time between the date of the initial ESD of the index lesion and the date of final visit to our hospital, the date of survival confirmed by the letter, or the date of death. In cases without ESD, the follow-up period was started from the date of the initial diagnosis of ESCC. Physical information, comorbidities, blood test data, and CT images within 6 months before the start of the followup period were evaluated as valid information. PMI was calculated by measuring the L3 level iliopsoas muscle area on CT images by the manual trace method.

\section{Post-Treatment Evaluation}

Curative resection was defined as epithelium (EP) and lamina propria mucosa (LPM) lesions with negative lymphovascular invasion and negative horizontal and vertical margins. Patients with curative resection, EP/LPM lesions with only positive horizontal margin, and muscularis mucosa (MM) lesions with negative lymphovascular invasion and negative horizontal/vertical margin among non-curative resections were categorized into the low-risk group of residual or recurrent cancer. Others were categorized into the high-risk group. The curative resection group was monitored 
Table 1. Characteristics of the patients and lesions

\begin{tabular}{|c|c|}
\hline & $N=241$ \\
\hline Age, median (range), years & $69.0(45-89)$ \\
\hline \multicolumn{2}{|l|}{ Sex, $n(\%)$} \\
\hline Male & $204(84.6)$ \\
\hline Female & $37(15.4)$ \\
\hline Follow-up period, median (range), months & $56(4-143)$ \\
\hline \multicolumn{2}{|l|}{ Location, $n(\%)$} \\
\hline Upper $(\mathrm{Ce}, \mathrm{Ut})$ & $38(15.8)$ \\
\hline Middle (Mt) & $105(43.6)$ \\
\hline Lower $(\mathrm{Lt}, \mathrm{Ae})$ & $98(40.7)$ \\
\hline \multicolumn{2}{|l|}{ Macroscopic type, $n(\%)$} \\
\hline Protruded & $6(2.5)$ \\
\hline Flat and depressed & $235(97.5)$ \\
\hline \multicolumn{2}{|l|}{ Treatment, $n(\%)$} \\
\hline ESD & $226(93.8)$ \\
\hline CRT & $5(2.1)$ \\
\hline Observation & $10(4.1)$ \\
\hline \multicolumn{2}{|l|}{ PMI, median (range), $\mathrm{cm}^{2} / \mathrm{mm}^{2}$} \\
\hline Male & $5.56(1.69-9.40)$ \\
\hline Female & $3.56(2.01-5.89)$ \\
\hline PNI, median (range) & $48.50(32.5-67.0)$ \\
\hline \multicolumn{2}{|l|}{ CONUT score, $n(\%)$} \\
\hline $0-1$ & $121(50.2)$ \\
\hline$\geq 2$ & $120(49.8)$ \\
\hline \multicolumn{2}{|l|}{ ECOG performance status, $n(\%)$} \\
\hline $0-1$ & $226(93.8)$ \\
\hline$\geq 2$ & $15(6.2)$ \\
\hline BMI, median (range) & $21.4(13.4-37.4)$ \\
\hline \multicolumn{2}{|l|}{ Charlson Comorbidity Index, $n(\%)$} \\
\hline 0 & $58(24.1)$ \\
\hline 1 & $28(11.6)$ \\
\hline 2 & $81(33.6)$ \\
\hline 3 & $45(18.7)$ \\
\hline 4 & $13(5.4)$ \\
\hline 5 & $5(2.1)$ \\
\hline$\geq 6$ & $11(4.6)$ \\
\hline Alcohol intake, median (range), g/day & $29(0-524)$ \\
\hline Brinkman index, median (range) & $740(0-3,000)$ \\
\hline
\end{tabular}

Ae, abdominal esophagus; Ce, cervical esophagus; CONUT, Controlling Nutritional Status; CRT, chemoradiotherapy; ECOG, Eastern Cooperative Oncology Group; ESD, endoscopic submucosal dissection; Lt, lower thoracic esophagus; Mt, middle thoracic esophagus; PMI, Psoas Muscle Index; PNI, Prognostic Nutritional Index; Ut, upper thoracic esophagus.

by esophagogastroduodenoscopy (EGD) every 6 months. Cases other than curative resection, including the CRT cases, were evaluated for recurrence by EGD at least twice a year and by CT scan at least once a year. Observation cases underwent EGD and CT scan as needed, such as when symptomatic.

\section{Statistical Analysis}

Quantitative data are expressed as median (range). OS was defined as the time from the starting date of follow-up to the date of
Table 2. Pathological characteristics

\begin{tabular}{ll}
\hline & $N=226$ \\
\hline $\begin{array}{l}\text { Tumor size, median (range), mm } \\
\text { Invasion depth, } n \text { (\%) }\end{array}$ & $20.0(5-80)$ \\
$\quad$ EP & $68(30.1)$ \\
LPM & $121(53.5)$ \\
MM & $25(11.1)$ \\
SM1 & $6(2.7)$ \\
SM2 & $6(2.7)$ \\
Horizontal margin, $n(\%)$ & $4(1.8)$ \\
$\quad$ Positive & $6(2.7)$ \\
Vertical margin, $n(\%)$ & $12(5.3)$ \\
$\quad$ Positive & $2(0.9)$ \\
Lymphatic invasion, $n(\%)$ & $183(81.0)$ \\
$\quad$ Positive & $43(19.0)$ \\
Vascular invasion, $n(\%)$ & additional therapy 15/43 \\
$\quad$ Positive & $206(91.2)$ \\
Curative resection, $n(\%)$ & $20(8.8)$ \\
Non-curative resection, $n(\%)$ & \\
Low risk, $n(\%)$ &
\end{tabular}

EP, epithelium; LPM, lamina propria mucosae; MM, muscularis mucosae; SM, submucosal layer. ${ }^{\text {a }}$ SM1 $\leq 200 \mu \mathrm{m}$ from MM. ${ }^{\text {b }}$ SM2 $>200 \mu \mathrm{m}$ from MM.

death from any cause or the date of the last survival confirmation. All survival data are shown with $95 \%$ confidence intervals (95\% CIs). The Kaplan-Meier method was used to evaluate OS rates, and the log-rank test was used to ascertain significance levels. ROC curve analysis was used to define the cutoff value for preference history, BMI, PMI, and CCI. The correlation between age and prognosis was evaluated using Spearman's correlation coefficient. The Cox proportional hazards model was used to calculate hazard ratios (HR). The items that were significant in the univariate analysis were used in the multivariate analysis. $p<0.05$ was considered statistically significant. The missing data were analyzed using pairwise deletion. All statistical analyses were performed with IBM SPSS version 25.0 (IBM Japan Ltd., Tokyo, Japan).

\section{Results}

\section{Patient and Lesion Characteristics}

A total of 241 patients were finally enrolled and analyzed. The patient and lesion characteristics are shown in Table 1. The median age of the population was 69.0 years. The median follow-up period was 56 months. The followup rates after 1, 2, and 3 years were 96.7\% (233/241), $86.7 \%$ (209/241), and 68.5\% (165/241), respectively. The male-to-female sex ratio was 6:1. The median PMI was 


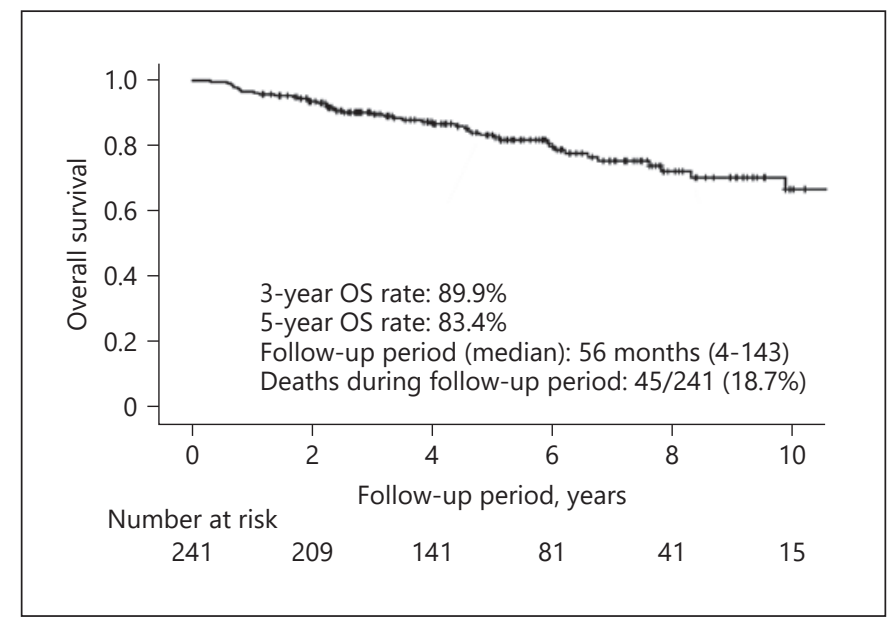

Fig. 1. The 3- and 5-year overall survival (OS) rates of all patients were 89.9 and $83.4 \%$, respectively. Deaths during the follow-up period were observed in 45/241 (18.7\%) cases, with 10 deaths from esophageal cancer and 35 deaths from other causes.

5.53 for men and 3.56 for women, and the median BMI was 21.4 , indicating a lean tendency.

\section{Pathological Result of ESD}

The pathological results obtained from ESD specimens are shown in Table 2. The rate of EP/LPM lesions was $83.6 \%$. The rates of positive horizontal margin, positive vertical margin, lymphatic invasion, and vascular invasion were $1.8,2.7,5.3$, and $0.9 \%$, respectively. There were 183 curative resections and 43 non-curative resections. Of the 43 non-curative resections, 15 patients received additional treatment, surgery, or CRT. Of the 226 ESD cases, 206 cases belonged to the low-risk group and the remaining 20 cases belonged to the high-risk group.

\section{Survival}

In all patients, the 3- and 5-year OS rates were 89.9 and $83.4 \%$, respectively. Deaths during the follow-up period were observed in $45 / 241$ (18.7\%) cases, with 10 deaths from ESCC and 35 deaths from other causes (Fig. 1). Causes of death in the enrolled patients are shown in Table 3. Of the deaths from other causes, 27 cases were death from other organ malignancy, and 3 cases were death from pneumonia. In the observation and CRT cases, no one died of esophageal cancer.

The optimal CCI cutoff value for predicting prognosis was defined as CCI $\leq 2$ and CCI $\geq 3$ using ROC curve analysis. The 3 - and 5-year OS rates in patients with CCI $\leq 2$ (96.2 and $91.5 \%$, respectively) were significantly higher than in those with CCI $\geq 3$ (75.4 and 64.8\%, respec-
Table 3. Causes of death in enrolled patients

\begin{tabular}{lc}
\hline & $N=45$ \\
\hline Deaths from esophageal cancer, $n(\%)$ & $10(22.2)$ \\
Deaths from other causes, $n(\%)$ & $35(77.8)$ \\
Other organ malignancy, $n(\%)$ & $27(60.0)$ \\
Pharyngeal cancer, $n(\%)$ & $9(20.0)$ \\
Lung cancer, $n$ (\%) & $6(13.3)$ \\
Hepatocellular carcinoma, $n(\%)$ & $4(8.9)$ \\
Gastric cancer, $n(\%)$ & $2(4.4)$ \\
Cholangiocarcinoma, $n(\%)$ & $2(4.4)$ \\
Pancreatic cancer, $n(\%)$ & $1(2.2)$ \\
Tongue cancer, $n(\%)$ & $1(2.2)$ \\
Malignant lymphoma, $n(\%)$ & $1(2.2)$ \\
Cancer of unknown primary, $n(\%)$ & $1(2.2)$ \\
Pneumonia & $3(6.7)$ \\
Urinary tract infection, $n(\%)$ & $1(2.2)$ \\
Cerebral infarction, $n(\%)$ & $1(2.2)$ \\
Aortic dissection, $n(\%)$ & $1(2.2)$ \\
Gastrointestinal bleeding, $n(\%)$ & $1(2.2)$ \\
Decrepitude, $n(\%)$ & $1(2.2)$ \\
\hline
\end{tabular}

tively, $p<0.001)$. ECOG PS was classified as PS $0-1$ and $\mathrm{PS} \geq 2$. The 3 - and 5 -year OS rates in patients with PS $0-1$ (91.9 and $85.1 \%$, respectively) were significantly higher than in those with PS $\geq 2$ (57.8 and $57.8 \%$, respectively, $p<0.001)$. PNI was classified into 2 groups, namely, PNI $<45$ and $\mathrm{PNI} \geq 45$. The 3 - and 5 -year OS rates in patients with PNI $\geq 45$ (92.5 and $87.0 \%$, respectively) were significantly higher than in those with PNI $<45$ (78.3 and $67.5 \%$, respectively, $p=0.001)$. The CONUT score was classified into 2 groups: normal CONUT $0-1$ and malnutrition CONUT $\geq 2$. The 3- and 5-year OS rates in patients with CONUT 0-1 (95.0 and $88.9 \%$, respectively) were significantly higher than in those with CONUT $\geq 2$ (84.2 and $77.4 \%$, respectively, $p=0.026$ ). Treatment options were classified into 3 groups: ESD group, CRT group, and observation group. The 3- and 5-year OS rates in patients in ESD group (95.1 and $89.0 \%$, respectively) were significantly higher than in those in CRT group ( 40.0 and $20.0 \%$, respectively, $p<0.001)$ and observation group ( 0 and $0 \%$, respectively, $p<0.001$ ) (Fig. 2a-e). No significant differences in prognosis rates were found among other study items.

\section{Prognostic Predictors for OS}

The prognostic predictors for OS are shown in Table 4. In the univariate analysis with the Cox proportional hazards model, ECOG PS $\geq 2$ (HR: 4.36; 95\% CI: 1.82-9.84; $p=0.001$ ), PNI < 45 (HR: 2.81; 95\% CI: $1.52-5.19 ; p=$ 0.001 ), CONUT $\geq 2$ (HR: 1.96; 95\% CI: $1.07-3.57 ; p=$ 0.028 ), and CCI $\geq 3$ (HR: 4.74; 95\% CI: $2.59-8.66$; $p<$ 


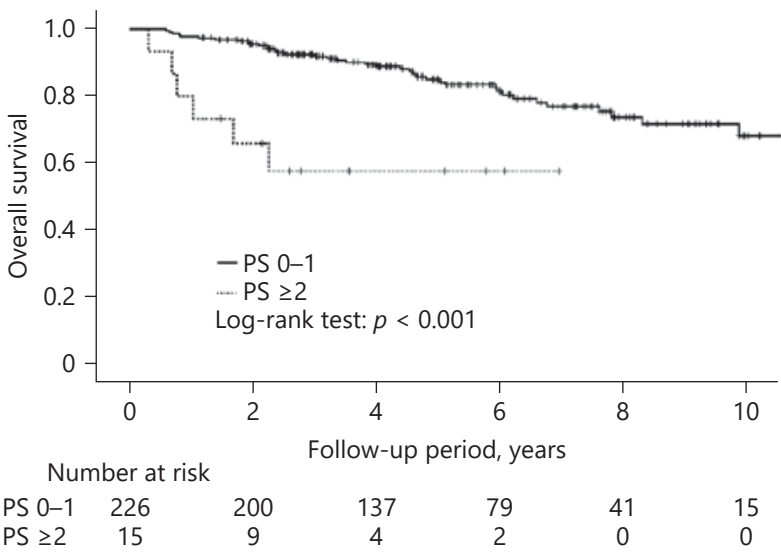

a

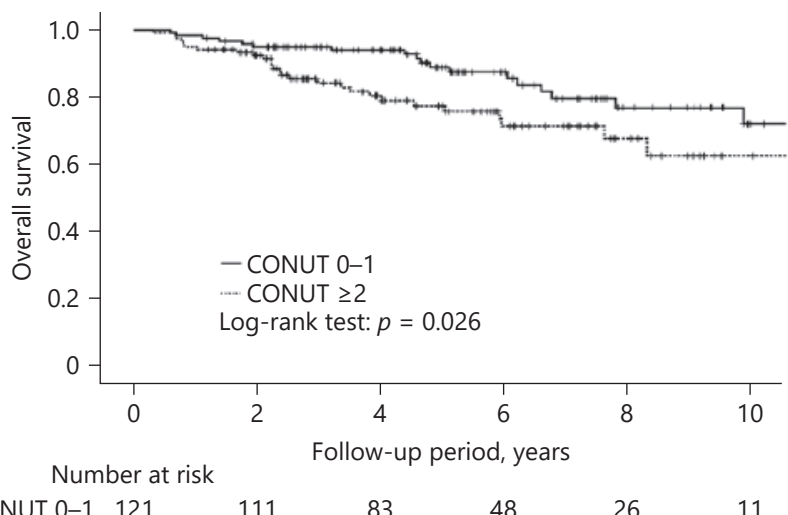

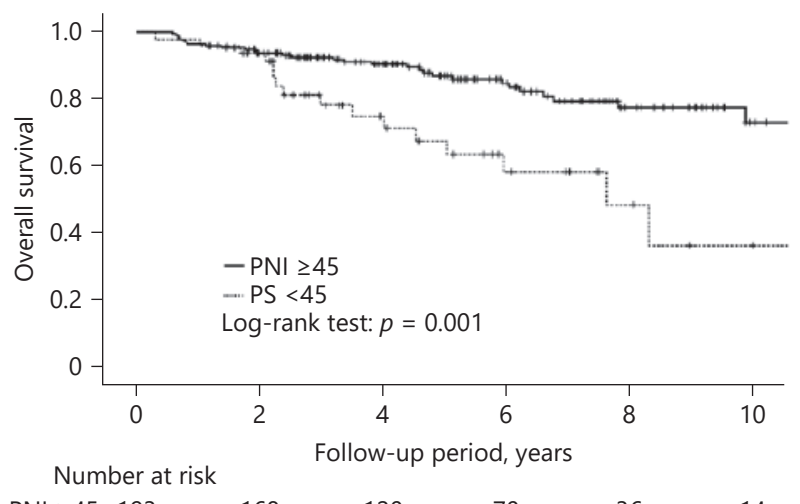

$\begin{array}{lllllll}\text { PNI } \geq 45 & 193 & 168 & 120 & 70 & 36 & 14\end{array}$ $\begin{array}{lllllll}\text { PS }<45 & 48 & 41 & 21 & 11 & 5\end{array}$

b

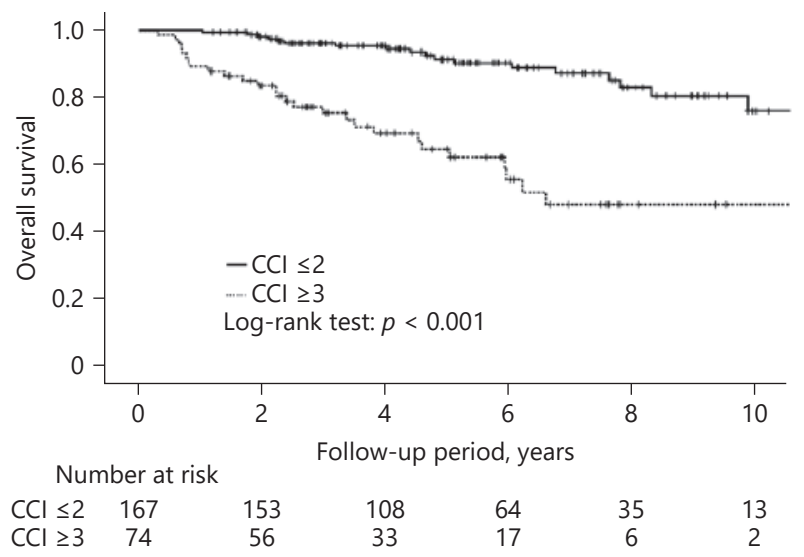

C

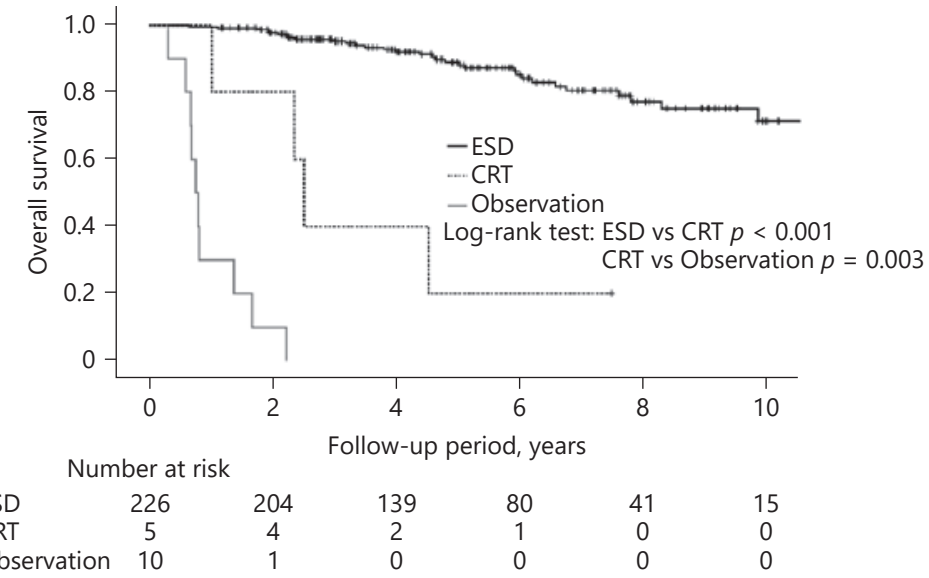

e

Fig. 2. a Overall survival (OS) rates comparing patients with Performance Status (PS) $0-1$ and $P S \geq 2$. b OS rates comparing patients with Prognostic Nutritional Index $(\mathrm{PNI}) \geq 45$ and $\mathrm{PNI}<45$. c OS rates comparing patients with Controlling Nutritional Status (CONUT) score $0-1$ and CONUT score $\geq 2$. d OS rates comparing patients with Charlson Comorbidity Index $(\mathrm{CCI}) \leq 2$ and $\mathrm{CCI} \geq 3$. e OS rates comparing patients with each treatment option: endoscopic submucosal dissection (ESD), chemoradiotherapy (CRT), and observation. 
Table 4. Prognostic predictors for overall survival

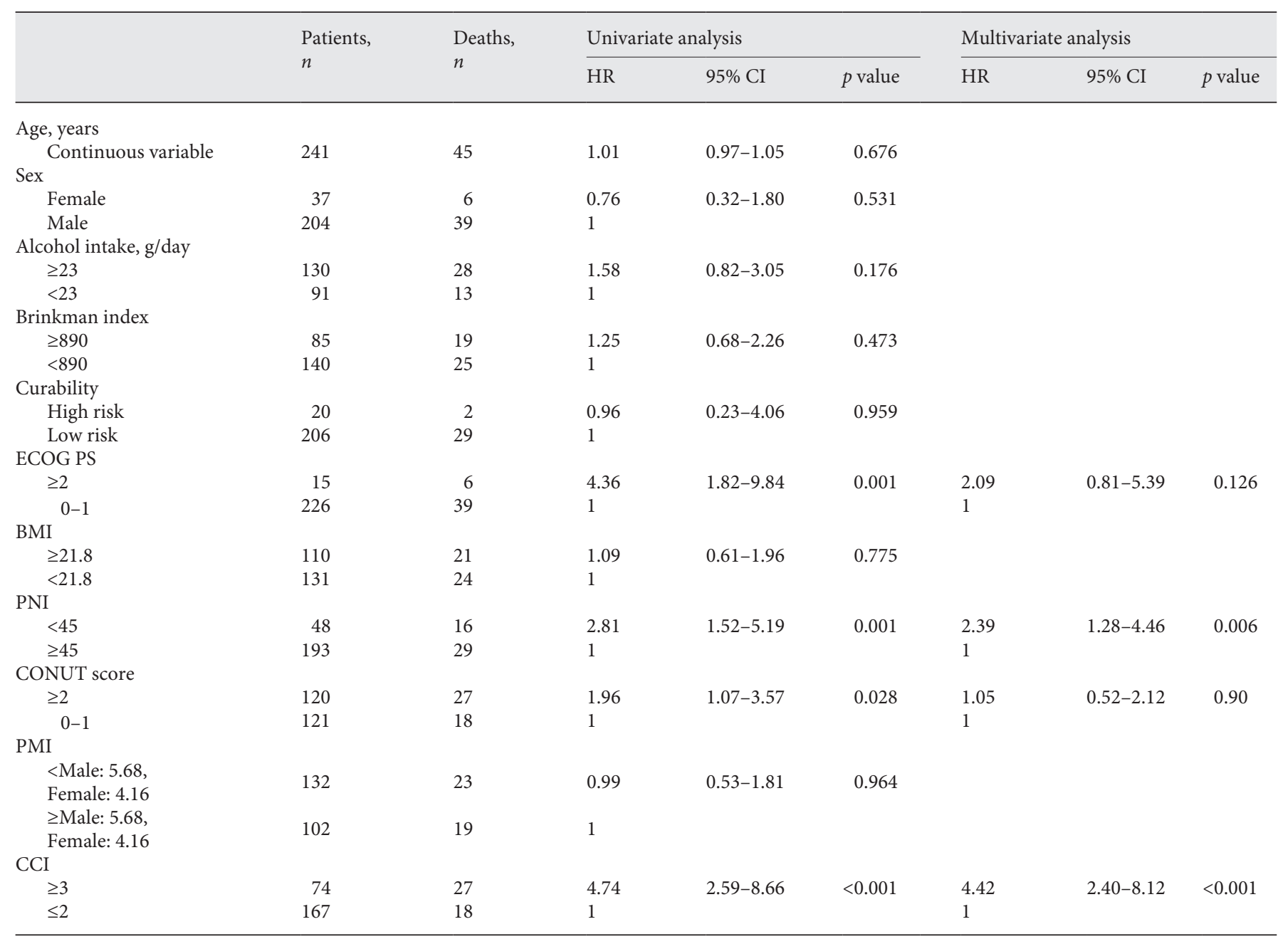

The $p$ value was calculated by Cox hazards regression analysis. CCI, Charlson Comorbidity Index; CI, confidence interval; CONUT, Controlling Nutritional Status; ECOG PS, Eastern Cooperative Oncology Group Performance Status; HR, hazard ratio; PMI, Psoas Muscle Index; PNI, Prognostic Nutritional Index.

$0.001)$ were significantly associated with OS. In the multivariate analysis, PNI < 45 (HR: 2.39; 95\% CI: 1.28-4.46; $p=0.006)$ and $\mathrm{CCI} \geq 3$ (HR, $4.42 ; 95 \% \mathrm{CI}, 2.40-8.12 ; p<$ $0.001)$ were significantly associated with OS. There was little correlation between age and prognosis.

We performed another analysis based on the results of the multivariate analysis. CCI was further divided into 3 groups: CCI 0-2, low CCI group; CCI 3-5, moderate CCI group; and CCI $\geq 6$, high CCI group. Based on the HR, $\mathrm{PNI} \geq 45$ was assigned 0 points, $\mathrm{PNI}<45$ was 1 point, and the low, moderate, and high CCI groups scored 0,2 , and 4 points, respectively. A score-based classification of $0-5$ points was created. The 3 - and 5-year OS rates in patients with a score 0 ( 97.7 and $93.3 \%$, respectively) were signifi- cantly higher than in those with a score 1 ( 88.0 and $81.7 \%$, respectively, $p=0.016)$ and a score $2(90.7$ and $80.5 \%$, respectively, $p=0.005)$. The 3 - and 5-year OS rates in patients with a score 3 (77.9 and $58.4 \%$, respectively) were significantly higher than in those with a score 4 (12.5 and $12.5 \%$, respectively, $p<0.001)$ and a score $5(0$ and $0 \%$, respectively, $p<0.001$ ) (Fig. 3).

\section{Discussion/Conclusion}

To the best of our knowledge, this is the first report to examine prognostic predictors including PNI, PMI, and CCI for superficial ESCC for which ESD was potentially 


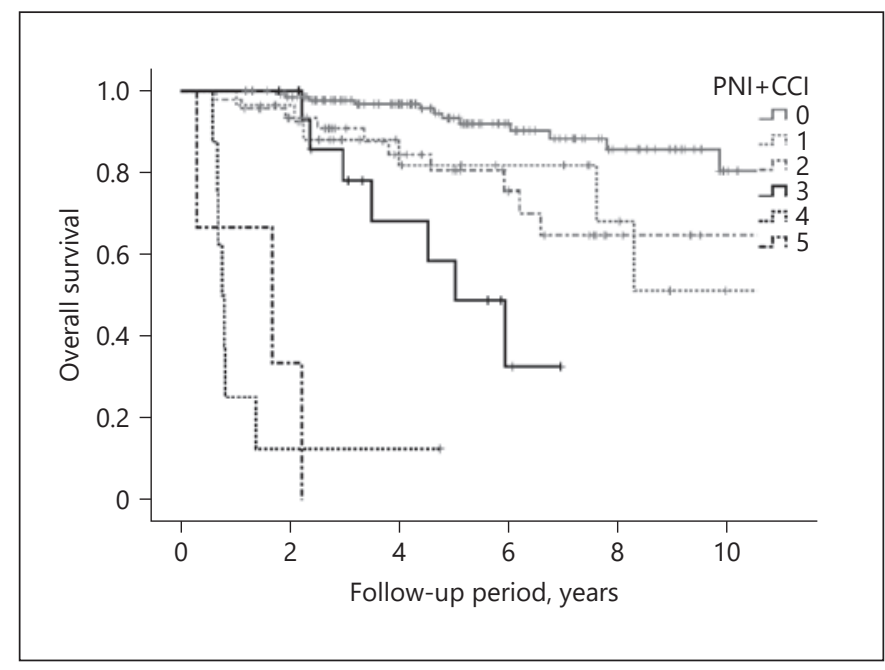

Fig. 3. Based on the hazard ratio, Prognostic Nutritional Index $(\mathrm{PNI}) \geq 45$ was assigned 0 points, $\mathrm{PNI}<45$ was 1 point, and the low, moderate, and high Charlson Comorbidity Index (CCI) groups scored 0,2 , and 4 points, respectively. A score classification of $0-5$ points was created. The 3 - and 5 -year overall survival (OS) rates in patients with a score 0 (97.7 and $93.3 \%$, respectively) were significantly higher than in those with a score $1(88.0$ and $81.7 \%$, respectively, $p=0.016)$ and a score $2(90.7$ and $80.5 \%$, respectively, $p=0.005)$. The 3 - and 5-year OS rates in patients with a score 3 (77.9 and $58.4 \%$, respectively) were significantly higher than in those with a score $4(12.5$ and $12.5 \%$, respectively, $p<0.001)$ and a score 5 ( 0 and $0 \%$, respectively, $p<0.001)$.

indicated. Studies on the prognostic predictors of ESD include reports that PNI, CCI, and PS were extracted as prognostic predictors for ESD of early gastric cancer for elderly patients [30]. In this study, PNI and CCI were finally extracted as prognostic predictors in the multivariate analysis. Therefore, we clarified that PNI and CCI are important prognostic predictors before ESD for ESCC. The Registration Committee for Esophageal Cancer of the Japan Esophageal Society reported that the 3- and 5-year OS rates after ESD treatment were 91.0 and $86.0 \%$, respectively [18]. Because no difference was found from the OS results of this study, our findings would be generally applicable to the whole Japanese cohort.

In this study, based on the $\mathrm{HR}, \mathrm{PNI} \geq 45$ was assigned 0 points, $\mathrm{PNI}<45$ was 1 point, and the low, moderate, and high CCI groups scored 0,2 , and 4 points, respectively. A score classification of $0-5$ points was created. In the highrisk group with scores 4 and 5 , that is, $\mathrm{CCI} \geq 6$, the 3 - and 5 -year OS rates were clearly low, at approximately $10 \%$. Most cases with CCI $\geq 6$ have comorbidities across multiple organs or solid cancer with distant metastasis. CCI is an index proposed in 1987 [14]. Medical progress has improved treatment outcomes for each disease, and an increase in controllable comorbidities and prognosis extension of solid cancer with distant metastasis have been achieved. Although the reconstruction of CCI may be necessary in the future, this study clarifies that the current CCI can be a sufficient prognostic predictor.

Guanrei et al. [31] reported that superficial ESCC progresses to advanced cancer in 4-5 years in the natural history. Until an esophageal tumor becomes advanced cancer, stenosis is unlikely to occur, with little influence on the patient's quality of life [32]. Observation without esophageal ESD is considered an accepted treatment strategy for patients at a high risk of death from other diseases within 3-4 years. Because the 3- and 5-year OS rates in the high-risk group with CCI $\geq 6$ were clearly low at approximately $10 \%$, observation strategy without ESD for superficial ESCC might be an acceptable option among patients with CCI $\geq 6$.

The PNI was originally designed to evaluate the nutritional and immunological status of patients undergoing surgical treatment for gastrointestinal diseases [33]. However, the original PNI required various parameters and was complicated to calculate. Therefore, Onodera et al. [1] proposed the modified PNI, which was calculated using the serum albumin level and lymphocyte count in the peripheral blood. They reported that the incidence of postoperative complications was higher in patients with a low PNI than in those with a high PNI [1]. Recently, studies have reported the importance of the PNI in the long-term outcomes of several malignancies [2-4]. The cutoff value is usually specified at 45 , since $\mathrm{PNI}<45$ is defined as moderate to severe malnutrition. In this study, the cutoff value of PNI was set to 45 , as previously reported, and was extracted as a significant prognostic predictor.

In this study, not only ESD cases but also lesions that were technically possible to undergo ESD including observation and CRT cases were included in the population. The clinical issue was the appropriateness of performing ESD at the time of diagnosis of superficial ESCC. Therefore, we aimed to evaluate the prognostic factors at the time of diagnosis of superficial ESCC potentially indicated for ESD: before selecting treatment option. Examining the comparison among the treatment options, the 3- and 5 -year OS rates in patients in ESD group were significantly higher than in those in CRT group and observation group. However, treatment options were not included in the examination items of the multivariate analysis in this study, because a strong selection bias was considered to have occurred during the selection of treatment method. In other words, CRT and observation were selected be- 
cause we considered that the prognosis would be clinically poor from the beginning at the time of selecting treatment method. In fact, all patients in CRT group had advanced pharyngeal cancer, and all in observation group had severe comorbidities. The prognosis of the group selected for observation was still poor; thus, this result might support the fact that the empirically established clinical selection was not significantly inappropriate.

In this study, we found little correlation between age and prognosis. The reported incidence of multiple primary cancers in patients with ESCC was $10-30 \%$, suggesting that even young patients have limited survival time due to comorbidities [21-23]. BMI and PMI were also examined, but they were not associated with prognosis. Patients with ESCC tend to be originally leaner, and some studies reported that increasing BMI can reduce the risk of ESCC [34, 35]. Average value -2 SD in PMI (male 6.36 , female 3.92) for healthy subjects aged $<50$ years has been proposed as the standard of low skeletal muscle mass in Japanese [8]. The median PMI in this study was 5.53 for men and 3.56 for women, and more than half of the subjects were judged to have low skeletal muscle mass. In other diseases, PMI has been reported to be useful as a prognostic predictor. Since sarcopenia was a fundamental factor for patients with ESCC, we considered that there was no association between PMI and prognosis.

This study had some limitations. First, this was a single-center retrospective study, and the number of cases was relatively small. In particular, there were few CRT cases and observation cases. Second, the treatment strategy for esophageal cancer was determined at the discretion of the attending physician and the patient. These facts may cause selection bias. However, in the observation cases and CRT cases, all the causes of death were the diseases other than esophageal cancer, so it appears that there was little association between treatment choice and prognosis. Therefore, PNI and CCI remain important prognostic predictors, and our study supports the importance of pre-treatment status. Third, since we have not examined physiological function tests including respiratory function tests in this study, there could be hidden prognostic factors.

In conclusion, CCI and PNI can be used as prognostic predictors of patients with superficial ESCC for which ESD is recommended. Moreover, observation strategy without ESD for superficial ESCC might be an acceptable option among patients with $\mathrm{CCI} \geq 6$, because this population is at a high risk of mortality from other causes prior to the appearance of symptoms associated with the progression of esophageal cancer.

\section{Statement of Ethics}

All study participants provided informed consent, and the study design was approved by the appropriate ethics review board of the Nagoya University Hospital (IRB No. 2015-0485).

\section{Conflict of Interest Statement}

The authors have no conflicts of interest to declare.

\section{Funding Sources}

The authors did not receive any funding.

\section{Author Contributions}

T.S. drafted and wrote the manuscript. The acquisition of the data was performed by K.F., E.I., T.S., K.M., T.Y., T.I., E.O., M.N., and H.K. T.S. analyzed the data. K.F. and M.F. revised the manuscript. M.F. gave the final approval of the manuscript.

\section{References}

1 Onodera T, Goseki N, Kosaki G. [Prognostic nutritional index in gastrointestinal surgery of malnourished cancer patients]. Nihon Geka Gakkai Zasshi. 1984 Sep;85(9): 1001-5.

2 Nozoe T, Ninomiya M, Maeda T, Matsukuma A, Nakashima H, Ezaki T. Prognostic nutritional index: a tool to predict the biological aggressiveness of gastric carcinoma. Surg Today. 2010 May;40(5):440-3.

3 Kanda M, Fujii T, Kodera Y, Nagai S, Takeda S, Nakao A. Nutritional predictors of postoperative outcome in pancreatic cancer. $\mathrm{Br} \mathrm{J}$ Surg. 2011 Feb;98(2):268-74.
4 Pinato DJ, North BV, Sharma R. A novel, externally validated inflammation-based prognostic algorithm in hepatocellular carcinoma: the prognostic nutritional index (PNI). Br J Cancer. 2012;106(8):1439-45.

5 Ignacio de Ulíbarri J, González-Madroño A, de Villar NG, González P, González B, Mancha A, et al. CONUT: a tool for controlling nutritional status. First validation in a hospital population. Nutr Hosp. 2005 Jan-Feb;20(1):38-45.

6 Kuroda D, Sawayama H, Kurashige J, Iwatsuki M, Eto T, Tokunaga R, et al. Controlling Nutritional Status (CONUT) score is a prognostic marker for gastric cancer patients after curative resection. Gastric Cancer. 2018 Mar; 21(2):204-12.

7 Yoshida N, Baba Y, Shigaki H, Harada K, Iwatsuki M, Kurashige J, et al. Preoperative nutritional assessment by controlling nutritional status (CONUT) is useful to estimate postoperative morbidity after esophagectomy for esophageal cancer. World J Surg. 2016 Aug;40(8):1910-7.

8 Hamaguchi Y, Kaido T, Okumura S, Kobayashi A, Hammad A, Tamai Y, et al. Proposal for new diagnostic criteria for low skeletal muscle mass based on computed tomography imaging in Asian adults. Nutrition. 2016 Nov-Dec;32(11-12):1200-5. 
9 Harada K, Suzuki S, Ishii H, Aoki T, Hirayama K, Shibata Y, et al. Impact of skeletal muscle mass on long-term adverse cardiovascular outcomes in patients with chronic kidney disease. Am J Cardiol. 2017 Apr;119(8):1275-80.

10 Okumura S, Kaido T, Hamaguchi Y, Fujimoto Y, Masui T, Mizumoto M, et al. Impact of preoperative quality as well as quantity of skeletal muscle on survival after resection of pancreatic cancer. Surgery. 2015 Jun;157(6): 1088-98.

11 Liu Y, Chen X, Wang Y, Wang F, Gong Y, Zhang J. Clinical features and prognostic factors for surgical treatment of esophageal squamous cell carcinoma in elderly patients. J Buon. 2019 May-Jun;24(3):1240-4.

12 Uemura M, Imataki O, Kawachi Y, Kawakami K, Hoshijima Y, Matsuoka A, et al. Charlson comorbidity index predicts poor outcome in CML patients treated with tyrosine kinase inhibitor. Int J Hematol. 2016 Nov; 104(5):6217.

13 Guo R, Yu W, Meng Y, Zhang K, Xu B, Xiao $\mathrm{Y}$, et al. Correlation of ASA grade and the Charlson comorbidity index with complications in patients after transurethral resection of prostate. Urology. 2016 Dec;98:120-5.

14 Charlson ME, Pompei P, Ales KL, MacKenzie CR. A new method of classifying prognostic comorbidity in longitudinal studies: development and validation. J Chronic Dis. 1987; 40(5):373-83.

15 Fujishiro M, Yahagi N, Kakushima N, Kodashima S, Muraki Y, Ono S, et al. Endoscopic submucosal dissection of esophageal squamous cell neoplasms. Clin Gastroenterol Hepatol. 2006 Jun;4(6):688-94.

16 Kim JS, Kim BW, Shin IS. Efficacy and safety of endoscopic submucosal dissection for superficial squamous esophageal neoplasia: a meta-analysis. Dig Dis Sci. 2014 Aug;59(8): 1862-9.

17 Tsujii Y, Nishida T, Nishiyama O, Yamamoto K, Kawai N, Yamaguchi S, et al. Clinical outcomes of endoscopic submucosal dissection for superficial esophageal neoplasms: a multicenter retrospective cohort study. Endoscopy. 2015 Sep;47(9):775-83.
18 Tachimori Y, Ozawa S, Numasaki H, Ishihara $\mathrm{R}$, Matsubara H, Muro K, et al. Comprehensive registry of esophageal cancer in Japan, 2010-2011. Esophagus. 2018 Jul;14(3):18952.

19 Iizuka T, Kikuchi D, Hoteya S. Outcomes of endoscopic submucosal dissection for superficial esophageal cancer in an elderly population: a retrospective single center cohort study. Endosc Int Open. 2019 Mar;7(3): E355-60.

20 Qi ZP, Chen T, Li B, Ren Z, Yao LQ, Shi Q, et al. Endoscopic submucosal dissection for early esophageal cancer in elderly patients with relative indications for endoscopic treatment. Endoscopy. 2018 Sep;50(9):839-45.

21 Nagasawa S, Onda M, Sasajima K, Takubo K, Miyashita M. Multiple primary malignant neoplasms in patients with esophageal cancer. Dis Esophagus. 2000;13(3):226-30.

22 Natsugoe S, Matsumoto M, Okumura H, Ish igami S, Uenosono Y, Owaki T, et al. Multiple primary carcinomas with esophageal squamous cell cancer: clinicopathologic outcome. World J Surg. 2005 Jan;29(1):46-9.

23 Otowa Y, Nakamura T, Takiguchi G, Yamamoto M, Kanaji S, Imanishi T, et al. Safety and benefit of curative surgical resection for esophageal squamous cell cancer associated with multiple primary cancers. Eur J Surg Oncol. 2016 Mar;42(3):407-11.

24 Kanda M, Koike M, Tanaka C, Kobayashi D, Hattori N, Hayashi M, et al. Modified systemic inflammation score is useful for risk stratification after radical resection of squamous cell carcinoma of the esophagus. Ann Surg Oncol. 2019 Oct;26(13):4773-81.

25 Nakajo K, Abe S, Oda I, Ishihara R, Tanaka M, Yoshio T, et al. Impact of the Charlson Comorbidity Index on the treatment strategy and survival in elderly patients after non-curative endoscopic submucosal dissection for esophageal squamous cell carcinoma: a multicenter retrospective study. J Gastroenterol. 2019 Oct;54(10):871-80.
26 Kitagawa Y, Uno T, Oyama T, Kato K, Kato $\mathrm{H}$, Kawakubo $\mathrm{H}$, et al. Esophageal cancer practice guidelines 2017 edited by the Japan Esophageal Society: part 1. Esophagus. 2019 Jan;16(1):1-24.

27 Kitagawa Y, Uno T, Oyama T, Kato K, Kato $\mathrm{H}$, Kawakubo H, et al. Esophageal cancer practice guidelines 2017 edited by the Japan esophageal society: part 2. Esophagus. 2019 Jan;16(1):25-43.

28 Yoshida T, Inoue $\mathrm{H}$, Usui S, Satodate $\mathrm{H}, \mathrm{Fu}-$ kami N, Kudo SE. Narrow-band imaging system with magnifying endoscopy for superficial esophageal lesions. Gastrointest Endosc. 2004 Feb;59(2):288-95.

29 Oyama T, Momma K. A new classification of magnified endoscopy for superficial esophageal squamous cell carcinoma. Esophagus. 2011;8:247-51.

30 Iwai N, Dohi O, Naito Y, Inada Y, Fukui A, Takayama S, et al. Impact of the Charlson comorbidity index and prognostic nutritional index on prognosis in patients with early gastric cancer after endoscopic submucosal dissection. Dig Endosc. 2018 Sep;30(5):616-23.

31 Guanrei Y, Songliang Q, He H, Guizen F. Natural history of early esophageal squamous carcinoma and early adenocarcinoma of the gastric cardia in the People's Republic of China. Endoscopy. 1988 May;20(3):95-8.

32 Short MW, Burgers KG, Fry VT. Esophageal cancer. Am Fam Physician. 2017 Jan;95(1): 22-8.

33 Buzby GP, Mullen JL, Matthews DC, Hobbs CL, Rosato EF. Prognostic nutritional index in gastrointestinal surgery. Am J Surg. 1980 Jan;139(1):160-7.

34 Abnet CC, Arnold M, Wei WQ. Epidemiology of esophageal squamous cell carcinoma. Gastroenterology. 2018 Jan;154(2):360-73.

35 Harada K, Ida S, Baba Y, Ishimoto T, Kosumi $\mathrm{K}$, Tokunaga R, et al. Prognostic and clinical impact of sarcopenia in esophageal squamous cell carcinoma. Dis Esophagus. 2016 Aug; 29(6):627-33. 